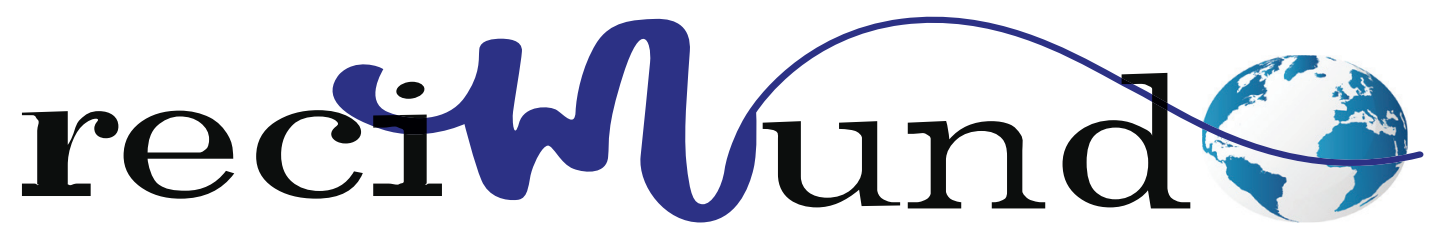

Revista Científica Mundo de la Investigación y el Conocimiento

DOI: 10.26820/recimundo/5.(4).oct.2021.12-21

URL: https://recimundo.com/index.php/es/article/view/1292

EDITORIAL: Saberes del Conocimiento

REVISTA: RECIMUNDO

ISSN: 2588-073X

TIPO DE INVESTIGACIÓN: Artículo de revisión

CÓDIGO UNESCO: 5801 Teoría y Métodos Educativos

PAGINAS: $12-21$

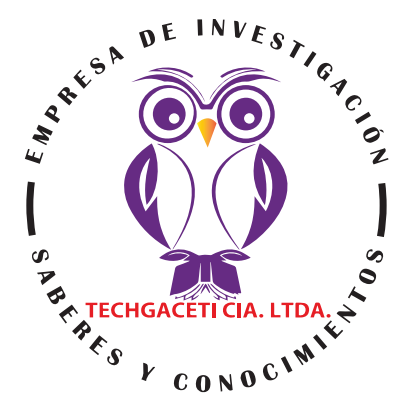

\title{
Universidad Virtual
}

Virtual University

Universidade Virtual

Denisse Salcedo Aparicio'; Erick Stalin Pazmiño Peñafiel2;

Vestalia Isabel Ceballos Muñoz3; Pedro Raúl Salcedo Aparicio4

\section{RECIBIDO: 02/09/2021 ACEPTADO: 20/09/2021 PUBLICADO: 30/10/2021}

1. Magister en Educación Informática; Licenciado en Ciencias de la Educación con Especialización en Informática; Psicóloga Clínica; Tecnólogo Pedagógico en Informática; Universidad de Guayaquil; Guayaquil, Ecuador; denisse.salcedoa@ ug.edu.ec; (iD https://orcid.org/0000-0002-2869-0977

2. Magister en Diseño y Gestión de Marca; Licenciado en Diseño y Producción Audiovisual; Universidad de Guayaquil; Guayaquil, Ecuador; erick.pazminop@ug.edu.ec; iD https://orcid.org/0000-0001-8493-1731

3. Magister en Docencia e Investigación Educativa; Licenciada en Ciencias de la Educación mención Educación Básica; Profesor de Segunda Enseñanza con Especialización en Lengua Española y Literatura; Investigador Independiente; Guayaquil, Ecuador; vestaliaceballos@yahoo.es; (D) https://orcid.org/0000-0002-4555-968X

4. Contador Público Autorizado; Ministerio de Educación; Ecuador; pedro.salcedo@educacion.gob.ec; (iD https://orcid. org/0000-0001-8877-4892

\section{CORRESPONDENCIA}

Denisse Salcedo Aparicio

denisse.salcedoa@ug.edu.ec

Guayaquil, Ecuador 


\section{RESUMEN}

En la actualidad, la oferta de la educación superior virtual ha ido en aumento en todo el mundo. Poco a poco se han integrado diferentes tecnologías en los procesos educativos, para poder ofrecer nuevas alternativas en el proceso de formación; en un principio como una herramienta dentro de la educación normal y presencial en las Instituciones de Educación Superior (IES), y que ha avanzado hasta poder tener programas completos de formación, avalados por estos institutos, e incluso contar con universidades virtuales completamente. La universidad virtual ofrece la posibilidad de formación, aprovechando los horarios flexibles, para personas que trabajan o mujeres embarazadas, y se presenta también como una oportunidad para migrantes y desplazados. El objetivo de la presente investigación es plasmar algunas generalidades acerca de la educación virtual universitaria. Entre estas generalidades de la educación virtual universitaria, se revisaron aspectos tales como: concepto, modelos, causas, universidad virtual y nuevas exigencias. El modelo de investigación es una revisión de tipo documental bibliográfico. Las principales modalidades de educación superior virtual son: E-Learning (aprendizaje electrónico), B-Learning, que combina el e-learning (encuentros asincrónicos) con encuentros presenciales (sincrónicos) y M-Learning (aprendizaje electrónico móvil). Los retos que presenta la Universidad virtual consisten básicamente en adaptar los modelos educativos universitarios con contenidos y principios filosóficos, pedagógicos, curriculares y didácticos contemporáneos propios de este momento tecnológico de la historia. La Universidad virtual rompe con los esquemas tradicionales educativos donde la tecnología es la herramienta de apoyo en la formación de futuros profesionales.

Palabras clave: Universidad, Virtual, Educación a distancia, Aulas virtuales, Campus virtuales.

\section{ABSTRACT}

Today, the supply of virtual higher education has been increasing around the world. Little by little, different technologies have been integrated into educational processes, in order to offer new alternatives in the training process; initially as a tool within normal and face-to-face education in Higher Education Institutions (IES), and which has progressed to have complete training programs, endorsed by these institutes, and even have fully virtual universities. The virtual university offers the possibility of training, taking advantage of flexible hours, for people who work or pregnant women, and is also presented as an opportunity for migrants and displaced persons. The objective of this research is to capture some generalities about virtual university education. Among these generalities of virtual university education, aspects such as: concept, models, causes, virtual university and new demands were reviewed. The research model is a bibliographic documentary type review. The main modalities of virtual higher education are: E-Learning (electronic learning), B-Learning, which combines e-learning (asynchronous encounters) with face-to-face (synchronous) encounters and M-Learning (mobile electronic learning). The challenges presented by the virtual university consist basically in adapting university educational models with contemporary philosophical, pedagogical, curricular and didactic content and principles typical of this technological moment in history. The virtual University breaks with traditional educational schemes where technology is the support tool in the training of future professionals.

Keywords: University, Virtual, Distance education, Virtual classrooms, Virtual Campus.

\section{RESUMO}

Hoje, a oferta de ensino superior virtual tem aumentado em todo o mundo. Aos poucos, diferentes tecnologias foram integradas aos processos educacionais, a fim de oferecer novas alternativas no processo de formação; inicialmente como uma ferramenta dentro da educação normal e presencial em Instituições de Ensino Superior (IES), e que tem progredido para ter programas de formação completos, endossados por esses institutos, e até ter universidades totalmente virtuais. A universidade virtual oferece a possibilidade de formação, aproveitando horários flexíveis, para pessoas que trabalham ou grávidas, e também se apresenta como uma oportunidade para migrantes e deslocados. O objetivo desta pesquisa é captar algumas generalidades sobre o ensino universitário virtual. Dentre essas generalidades da formação universitária virtual, foram revisados aspectos como: conceito, modelos, causas, universidade virtual e novas demandas. O modelo de pesquisa é uma revisão bibliográfica do tipo documental. As principais modalidades de ensino superior virtual são: E-Learning (aprendizagem eletrónica), B-Learning, que combina e-learning (encontros assíncronos) com encontros presenciais (síncronos) e M-Learning (aprendizagem eletrónica móvel). Os desafios apresentados pela universidade virtual consistem basicamente em adaptar modelos de formação universitária com conteúdos e princípios filosóficos, pedagógicos, curriculares e didáticos contemporâneos próprios deste momento tecnológico da história. A Universidade virtual rompe com os esquemas educacionais tradicionais onde a tecnologia é a ferramenta de apoio na formação dos futuros profissionais.

Palavras-chave: Universidade, Virtual, Educação a distância, Salas de aula virtuais, Campus virtual. 


\section{Introducción}

El acceso a la educación superior es una necesidad global que ha originado cambios en las modalidades educativas, las cuales van evolucionado constantemente para adaptarse a los requerimientos de la sociedad moderna. La incorporación de las tecnologías de información y comunicación (TIC) a la educación han hecho posible el cambio de los paradigmas de gestión del conocimiento de las escuelas tradicionales centradas en la enseñanza hacia la educación centrada en el aprendizaje del estudiante. (Fernández \& Vallejo, 2014, p. 30)

La manera en la que se planifica, diseña e implementa un curriculum universitario con el propósito de lograr los objetivos de enseñanza y aprendizaje, se llaman modalidades. La Educación Presencial y a Distancia son las dos modalidades mayormente empleadas por las universidades para desarrollar su curriculum. No obstante, para algunos autores la Educación Virtual está logrando un importante posicionamiento ya que esta modalidad distribuye el tiempo de estudio de un modo más flexible que la presencialidad y ofrece un proceso educativo más intenso e interactivo en el sentido que proporciona un mayor tiempo de relación profesor y alumno. (Durán Rodríguez, 2015, p. 47)

Fernández \& Vallejo, (2014) destaca algunas de las ventajas de la educación virtual universitaria:

La educación virtual ofrece la posibilidad de formación, aprovechando los horarios flexibles, para personas que trabajan o mujeres embarazadas, y se presenta también como una oportunidad para migrantes y desplazados. En suma, la incorporación de perspectivas de género, de discapacidades, de las culturas indígenas, de la pluralidad cultural y la pertinencia global se constituyen como los elementos relevantes para promover la renovación, actualización y adaptación de las universidades a los requerimientos de la sociedad moderna. (34)

Actualmente, con la pandemia de Covid-19, la cual representa un reto enorme para la educación, el sistema educativo virtual ha sido la solución en la prosecución de la educación a nivel global y ha conllevado al crecimiento de la oferta de la universidad virtual. Cóndor-Herrera, 2020, citado por Quezada, (2021) manifiesta que a partir de la pandemia se decretaron medidas de distanciamiento social y confinamiento, que llevaron a la población a aislarse en sus hogares para evitar el contagio. Asimismo, citando a la CEPAL, 2020 destacan lo siguiente:

El mayor impacto lo tienen las actividades que dependen del contacto interpersonal, se forzó una rápida virtualización de estas actividades, entre ellas la educación, para el 20 de marzo se habían suspendido las clases en la mayoría de los países de Latinoamérica, forzando a la educación a encontrar alternativas para continuar con sus labores a la distancia, fue entonces que las TIC se convirtieron en un recurso indispensable para continuar con esa modalidad de educación. (p. 156)

En tal sentido, El propósito fundamental del presente estudio es plasmar algunas generalidades acerca de la educación virtual universitaria. Entre estas generalidades de la educación virtual universitaria, se revisaron aspectos tales como: concepto, modelos, causas y universidad virtual.

\section{Materiales y métodos}

La realización del presente estudio requirió una metodología de tipo documental o bibliográfica, ya que para su desarrollo fueron necesarios equipos tales como ordenadores con conexión a internet, por medio de los cuales se llevó a cabo una búsqueda de material bibliográfico digitalizado. 
Para ubicar el material se usaron buscadores como Google y Google Académico. Igualmente, fue de gran utilidad el portal de la Organización de las Naciones Unidas para la Educación, la Ciencia y la Cultura UNESCO.

El material base del estudio consistió en informes, protocolos, tesis de grado y otras clases de contenidos. Contenidos repetidos, las editoriales o cartas editoriales, anotaciones académicas y todo documento carente de sustento científico o bajo nivel de evidencia fueron excluidos de la selección.

La investigación se desarrolló durante el mes de septiembre de 2021. Los descriptores para la búsqueda fueron los siguientes: "Universidad virtual", "educación virtual universitaria"; "modelos de educación virtual universitaria", "Campus virtual". La información resultante se filtró bajo los criterios de idioma español. Posteriormente, fueron seleccionados aquellos trabajos que tuvieran mayor relevancia y con correlación del tema. Por último, se seleccionaron aquellos trabajos de más actualidad (fecha de publicación: 2012 - 2021).

El equipo investigador manifiesta que no tener conflicto de intereses.

\section{Resultados}

\section{Educación Virtual}

Torres (2003), citado por Leal Hoyos, (2020) plasma el siguiente concepto amplio de educación virtual:

La educación virtual se concibe como un sistema abierto y permanente fundamentado en un nuevo enfoque pedagógico que favorece el estudio autónomo e independiente del estudiante; que propicia, con la ayuda de un cuerpo de tutores profesionales, la autogestión formativa, el trabajo en equipo en el ciberespacio, la generación de procesos interactivos académicos, mediados por una acción dialógica: estudiante-estudiante y tutor-estudiante, con soportes tecnológicos y de comunicación avanzada, con actividades académicas diseñadas para ser realizadas tanto en el interior como al exterior del campus universitario, con el fin de que cada vez más jóvenes y profesionales tengan acceso al conocimiento y a la actualización de los saberes. (p. 52)

\section{Modelos de Educación Virtual}

Los modelos educativos universitarios aparecen como elementos impulsores de la calidad de la formación profesional, los que actúan como documentos rectores de la actividad docente y estudiantil, con orientaciones de nivel filosófico, pedagógico, curricular y didáctico, actualizadas y coherentes con los requerimientos demandados por la sociedad, y que dan sentido a los procesos y resultados académicos de la universidad. Los modelos educativos universitarios contienen principios filosóficos, pedagógicos, curriculares y didácticos contemporáneos propios de cada momento y espacio histórico. Algunos valoran más algunos componentes que otros, no obstante, en todos se visualiza globalmente como una educación que se propaga social y económicamente mediante la digitalización e innovación. Si bien la mayoría de los modelos fueron elaborados pensando en su aplicación en la educación presencial, contienen una mirada de una formación a distancia y virtual, para un futuro que sus autores consideraban de largo plazo, planificando en primer lugar implementar de manera progresiva las herramientas tecnológicas durante la formación académica. (Hidalgo, 2021, p. 9, 10)

Actualmente, se vive una época de grandes transformaciones sociales, económicas, culturales y tecnológicas, donde se dan nuevas oportunidades y grandes desafíos a causa del avance vertiginoso de las tecnologías, las cuales han adquirido un significado preponderante, y aunque a través de ellas se tenga la posibilidad de acceder fácilmente a la información, en ocasiones

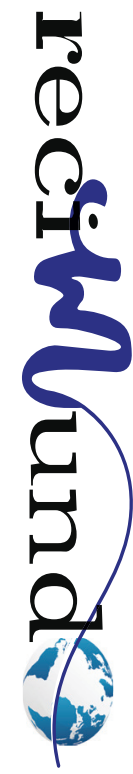


ésta es difícil de asimilar, aunado a que el tiempo se convierte en recurso escaso y costoso, siendo imprescindible la selección y organización de ésta información para su mejor entendimiento. Existen diferentes tipos de modalidades que se pueden utilizar en la Educación Virtual y que tienen la finalidad de cambiar el modelo tradicional de Educación (ver Figura 1). Para (Guerrero, Rojas, \& Villafañe, 2019) entre las principales, destacan:

- E-Learning: La entrega de educación a través de la Tecnología de la Información y la Comunicación (ITC) utilizando una amplia variedad de diseños y formatos de instrucción, e incluye aprendizaje sincrónico y asíncrono. el cual ofrece una variedad de modos de aprendizaje que mejoran el conocimiento y el rendimiento de los estudiantes y es de gran utilidad, dado que brinda flexibilidad de tiempo y espacio, el estudiante puede controlar los contenidos, su ritmo de aprendizaje de acuerdo a sus necesidades, brinda más oportunidad para acceder a la educación, aumenta la calidad del contenido instruccional, entre otros.
- B-Learning: Blended learning es un "diseño docente en el que tecnologías de uso presencial (físico) y no presencial (virtual) se combinan con objeto de optimizar el proceso de aprendizaje". A través de este modelo, se plantea una formación o enseñanza mixta, en el que el educador toma un rol tradicional, pero al mismo tiempo aprovecha las posibilidades que le brinda las plataformas virtuales, dando espacio a un entorno educativo que favorece el quehacer docente; de este modo, logra ejercer su función como un educador tradicional y un tutor a distancia.

- M-Learning: Es un modelo de formación que utiliza dispositivos móviles para el aprendizaje. Las tecnologías móviles han redefinido el panorama educativo, pues aportan características propias de los dispositivos móviles como movilidad, conectividad, ubicuidad y permanencia, que son necesarias en los sistemas de educación a distancia. (p. 19. 34-37)

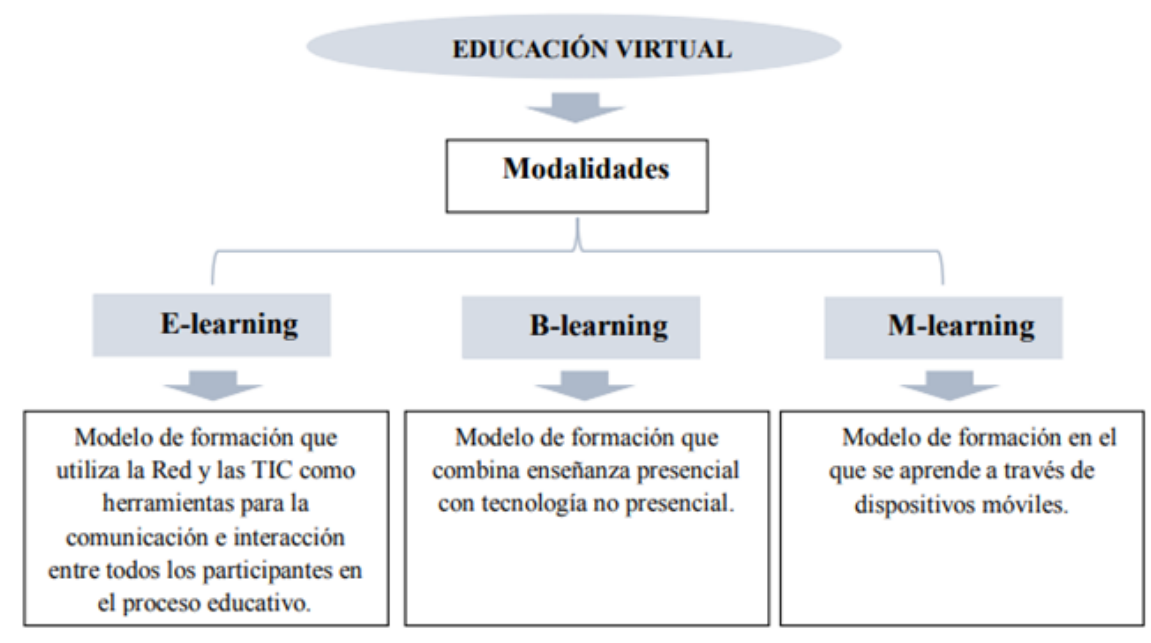

Figura 1. Mapa conceptual. Modalidades de la Educación Virtual

Nota. "Impacto de la Educación Virtual en Carreras de Pregrado del Área de Ciencias de la Salud. Una Mirada de las Tecnologías Frente a la Educación". Guerrero, Rojas, \& Villafañe. (2019)

Recuperado de: https://repository.ucc.edu.co/bitstream/20.500.12494/14845/3/2019_ impacto_educacion_virtual.pdf 


\section{Causas del Aumento de la Educación Vir- tual Universitaria}

Existen diversas causas que justifican el aumento de la Universidad Virtual. Al respecto, Salgado García, (2015) acota lo siguiente:

- Por un lado, las mismas familias demandan más acceso a educación, debido a que los salarios son insuficientes y la educación se percibe como el mecanismo de movilidad social por excelencia. Estas familias son de clases medias-bajas, dado que las clases más acomodadas, por lo general pueden invertir más en educación superior y enviar a sus hijos a las universidades públicas y privadas de prestigio, las cuales son esencialmente presenciales.

- Los adultos requieren de oportunidades de capacitación permanente para adquirir nuevas habilidades, en el contexto de competencia por puestos de trabajo mejor remunerados; las personas que trabajan en zonas rurales, sobre todo docentes $u$ otros, no pueden acceder a la educación superior en las ciudades por razones de localización geográfica; las mujeres demandan educación, pero deben atender a sus hijos; los jóvenes deben trabajar para aumentar los ingresos familiares y no pueden estudiar en universidades presenciales con horarios definidos.

- Por otra parte, hay una tendencia a reducir costos en el Estado, producto de políticas de reducción de gasto público. Si bien la educación superior virtual de alta calidad puede ser igual o más costosa que la presencial, lo que se ha venido haciendo es abrir opciones de menor calidad y menor costo, dirigidas a las masas.

- Asimismo, las universidades están adoptando un "modelo corporativo", viéndose a sí mismas como empresas en busca de captar clientes. En este sentido, las tecnologías están ayudando a trascender fronteras y a regionalizar sus ofertas no solo dentro de los países, sino también a escala transnacional. Universidades de España, Chile, el Reino Unido, Estados Unidos, Canadá y México, publicitan regularmente programas virtuales o semi presenciales en toda Latinoamérica.

- La oferta virtual está además sacando partido del relativo estancamiento de la financiación estatal a las universidades públicas. El Estado ha disminuido su participación, dejando así espacio para una creciente inversión privada en educación superior. Asimismo, en los tratados de libre comercio se ha incluido la educación como un "bien de consumo", que permite desregular la entrada de "corporaciones universitarias" de carácter transnacional en los diferentes países de América Latina. (p. 142, 143)

\section{Universidad Virtual}

En los países de Latinoamérica la inclusión de las TIC en los procesos educativos, en la formación en educación superior, ha significado un reto que varía en cada país, según los intereses políticos y económicos que se tengan, y sobre todo por el nivel de desarrollo científico y tecnológico que es diferenciado según las condiciones sociales de los mismos. Poco a poco se han integrado diferentes tecnologías en los procesos educativos, para poder ofrecer nuevas alternativas en el proceso de formación; en un inicio como una herramienta dentro de la educación normal y presencial en las Instituciones de Educación Superior (IES), y que ha avanzado hasta poder tener programas completos de formación, avalados por estos institutos, e incluso contar con universidades virtuales completamente. (Victorino, 2012)

Para Palacios, Rodríguez, \& Forero, (2015) esta nueva dinámica de las tecnologías adaptadas al sistema de educación puede generar un cambio en los paradigmas tradicionales de la educación y en los procesos administrativos y académicos de las institu-

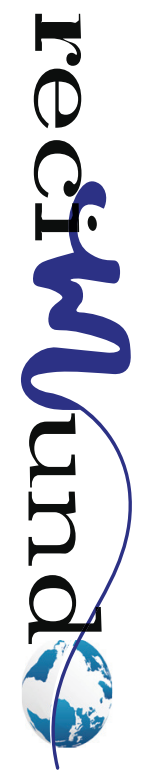


ciones de educación superior (IES), dando origen a los conocidos ambientes virtuales de aprendizaje que llevan a la construcción del campus virtual, en el que convergen servicios académicos y administrativos para la comunidad. "Las Universidades virtuales son aquellas que tienen programas presenciales y generan una estructura organizacional virtual (universidad virtual) en interdependencia con la presencial. Cuenta con programas totalmente virtuales". (p. 98)

Ortiz, 2007 citado por Urbina \& Salinas, (2014) acerca del campus virtual manifiesta lo siguiente:

El campus virtual designa distintos sitios web a disposición de una comunidad educativa, con la facultad de proveer recursos pedagógicos y funcionalidades de comunicación y de interacción. Un campus virtual puede entenderse como una metáfora o correlato del campus físico que asimila sus elementos a un ambiente virtual, y cuya recreación se logra mediante distintas aplicaciones informáticas y tecnologías web. Son, en definitiva, el intento de situar un campus universitario en el marco de la virtualidad, de forma que permita a los estudiantes acceder a la docencia, a la organización de la misma -aulas, matrícula, etc-y a los demás espacios complementarios como la biblioteca, los servicios universitarios, etc. Asimismo, es importante destacar que existen modelos de campus virtuales o de experiencias de uso de las TIC en la formación online en el nivel superior. En todos ellos puede comprobarse que ofrecen distintos grados de evolución, que en gran medida se traduce en el nivel de complejidad y madurez del sistema. (p. 2)

Para Fariña, González, \& Area, (2013) en la universidad virtual, los profesores y estudiantes universitarios cuentan de esta forma con una extensión de las aulas presenciales en las aulas virtuales, que permiten nuevas formas de aprender autónomamente y colaborativamente. La incorporación de las aulas virtuales a la docencia permite contar con un espacio en el que ofrecer diferentes herramientas a los estudiantes: información, foros para la comunicación e interacción, así como tareas y actividades fácilmente evaluables. Concretamente los docentes a través de un aula virtual pueden:

- Gestionar contenidos e información: pueden presentar al alumnado los apuntes de la asignatura en formato textual, pero además pueden ofrecer presentaciones multimedia, imágenes, gráficas, esquemas, vídeos, enlaces de interés.

- Ofrecer recursos Web 2.0: pueden insertar en las aulas virtuales, a través de código embebido, otros objetos de aprendizaje como Blogs, Webquest, redes sociales.

- Favorecer la comunicación: pueden crear foros de novedades, foros de discusión, foros de dudas, son espacios que promueven el aprendizaje cooperativo entre los estudiantes. Además, cuentan con herramientas para realizar tutorías individuales y grupales.

- Solicitar tareas-actividades: pueden solicitar al alumnado la realización de diferentes tareas que permitan desarrollar capacidades y competencias. Los alumnos pueden subir archivos o enlazar los trabajos que se encuentren en otros espacios web, pueden desarrollar Wikis de manera colaborativa, etc. Además de contar con herramientas de evaluación y autoevaluación.

- Evaluación del aprendizaje: los docentes cuentan con herramientas para evaluar los trabajos del alumnado, siendo muy importante para el aprendizaje el FeedBack que se puede establecer entre profesores y alumnos. (p. 2)

\section{Nuevas exigencias}

En la actualidad, el volumen de recursos educativos digitales impone nuevas exigencias a los sistemas e instituciones de la educación superior, en lo relativo al desarrollo 
de los programas educativos, los planes de estudio y los procesos de aprendizaje novedosos e innovadores, así como a las vías de acceso a la enseñanza superior.

Todo lo anteriormente mencionado propiciado por la existencia de modelos de prestación de servicios de aprendizaje en línea, a distancia, de educación abierta, mixta y cursos de corta duración, basados en la adquisición de competencias tales como los Cursos en línea masivos y de libre acceso (MOOC) y los Recursos Educativos Abiertos (REA). El enorme potencial que propicia el aprendizaje en línea en general, y en particular, bajo la forma de MOOC, abre nuevas vías de acceso a la enseñanza superior, así como al aumento de las posibilidades de educación mediante soluciones alternativas flexibles. En tal sentido, se recomienda que los gobiernos formulen políticas y programas para brindar una educación superior a distancia de calidad, adecuadamente financiada, basada en las tecnologías, en particular mediante cursos en línea masivos y de libre acceso que responden a las normas de calidad a fin de mejorar la accesibilidad a éstos. (Organización de las Naciones Unidas para la Educación, la Ciencia y la Cultura - UNESCO, 2021)

Inga \& Aguirre, (2021) en su estudio: el enfoque de la educación virtual desde una perspectiva holístico frente a la pandemia del COVID-19, cuyo propósito fue el de determinar en qué medida el enfoque de la educación virtual cumple con impartir un aprendizaje holístico en estudiantes universitarios, llegaron a las siguientes conclusiones:

En la educación digital, de acuerdo a la experiencia empírica, es necesario planificar las acciones que se va desarrollar, durante las clases no presenciales (el contenido, interacción, actividades de aprendizaje, evaluación y las herramientas tecnológicas), con el fin de impulsar un aprendizaje activo, donde el estudiante se convierte en constructor de su propio aprendizaje (genere ideas, discute, argumenta, gestiona aprendizaje con autonomía, construye, crea, genera conocimiento, soluciona problemas, etc.). Para ello es imprescindible tender redes de comunicación (sincrónica, asincrónica), entre el docente y estudiante, así como también, estar en constante renovación con las estrategias y técnicas actuales. El objetivo a futuro es impulsar un espacio de aprendizaje seguro, activo con las herramientas digitales adecuadas que incorporé al estudiante a la investigación (buscar conocimientos basado en la innovación, formular opiniones con ideas originales, promover el pensamiento divergente y asunción de riesgos), a la responsabilidad social (Impulsar el autocontrol, la actitud ética, proactiva, y visión inclusiva), y al liderazgo (liderar equipos disciplinarios o multidisciplinarios, demostrar empatía, respeto, etc.), con la intención de prepararlos, para enfrentar a un mundo globalizado, que con pasos agigantados incorpora al ser humano a la competitividad y a la meritocracia. Asimismo, el compromiso de las autoridades de los estamentos universitarios, deben garantizar la capacitación en competencias virtuales a los docentes, para que puedan convertirse en residentes virtuales y proporcionar a los estudiantes una excelencia formación académica. (p. 93, 94)

Por su parte, Hidalgo, (2021) en las conclusiones de su estudio acerca de los modelos educativos en la educación virtual universitaria, destaca:

Se hace necesario reflexionar sobre los cambios que deben ser introducidos en los modelos educativos universitarios, por ejemplo: la adecuación de los planes curriculares y una didáctica específica para la educación virtual, así también criterios para la selección y diseño de recursos virtuales y una propuesta de evaluación para la educación remota. (p. 10) 
Juca Maldonado, (2016) destaca que una nueva exigencia para los profesionales de la educación virtual es la formación continua. La sociedad de la información y el conocimiento, demandan cada vez más el aprendizaje continuo a lo largo de la vida. Este autor en su estudio, revisa los elementos que contribuyen a pensar la formación docente como una articulación de factores que, sin negar los contextos, permiten la apropiación de los recursos de la tecnología digital para la enseñanza, de lo resultados concluye que es necesario:

Es importante conocer los requisitos para la formación del docente innovador, es fundamental generar los espacios de reflexión para superar barreras o favorecer la implementación de prácticas correctas para la innovación en los programas de educación a distancia, combinando las TIC e Internet. Entre las diversas medidas a tomar se hallan las inversiones tecnológicas de infraestructura y de redes, con soportes técnicos constantes, para una buena administración y organización de las propuestas curriculares de formación. Aprender a aprender de un modo autorregulado (metacognitivo, que implica fortificar la autonomía de la persona que aprende) en situaciones combinadas presenciales y virtuales. Hacia una práctica profesional reflexiva del profesor. (p. 111)

\section{Conclusiones}

Las TICS juegan un papel fundamental en las relaciones entre estudiantes y docentes e información y conocimiento, los cuales a su vez forman parte de los procedimientos de aprendizaje y enseñanza. En consecuencia, resulta imperioso que tanto docentes, como estudiantes, personal administrativo y todo el personal de la institución superior reorienten su paradigma de educación al contemplar alternativas novedosas en la construcción del conocimiento, el manejo de la información y en la gestión de los procesos administrativos y los recursos.
En tal sentido, la universidad virtual pone al alcance de estudiantes, docentes, demás personal de la institución, interesados en general, incluso a otras instituciones la posibilidad de acceder a la información, gestiones, aulas virtuales y un sinfín de servicios, según la disponibilidad de tiempo y sin importar el espacio.

Si bien es cierto que a nivel global existen desigualdades en cuanto al acceso y disponibilidad de las tecnologías, no es menos cierto que cada vez más esa barrera se va rompiendo y que la virtualización de la educación superior representa nuevas formas de aprender autónomamente y colaborativamente.

Los retos que presenta la Universidad virtual consisten básicamente en adaptar los modelos educativos universitarios con contenidos y principios filosóficos, pedagógicos, curriculares y didácticos contemporáneos propios de este momento tecnológico de la historia. La Universidad virtual rompe con los esquemas tradicionales educativos donde la tecnología es la herramienta de apoyo en la formación de futuros profesionales.

\section{Bibliografía}

Durán Rodríguez, R. (2015). La Educación Virtual Universitaria como medio para mejorar las competencias genéricas y los aprendizajes a través de buenas prácticas docentes. Tesis Doctoral, Universidad Politécnica de Catalunia, Programa de Doctorado de Ingeniería de Proyectos: Medio Ambiente, Seguridad, Calidad y Comunicación, Barcelona. Recuperado el 15 de Septiembre de 2021, de https://www.tdx.cat/bitstream/handle/10803/397710/TRADR1de1.pdf

Fariña, E., González, C., \& Area, M. (2013). ¿Qué uso hacen de las aulas virtuales los docentes universitarios? RED, Revista de Educación a Distancia, 35, 1-3. Recuperado el 27 de Septiembre de 2021, de https://www.um.es/ead/red/35/farina_et_al.pdf

Fernández, K., \& Vallejo, A. (2014). La educación en línea: una perspectiva basada en la experiencia de los países. Revista de Educación y Desarrollo, 29-39. Recuperado el 05 de Septiembre de 2021, 
de https://www.cucs.udg.mx/revistas/edu_desarrollo/anteriores/29/029_Fernandez.pdf

Guerrero, A., Rojas, C., \& Villafañe, C. (2019). Impacto de la Educación Virtual en Carreras de Pregrado del Área de Ciencias de la Salud. Una Mirada de las Tecnologías Frente a la Educación. Tesis Doctoral, Universidad Coopertiva de Colombia, Facultad de Educación, Bogotá. Recuperado el 02 de Septiembre de 2021, de https://repository.ucc. edu.co/bitstream/20.500.12494/14845/3/2019_impacto_educacion_virtual.pdf

Hidalgo, L. (2021). Modelos educativos en la educación virtual universitaria. Revista de investigación de la Facultad de Humanidad, 9(1), 4-13. Recuperado el 25 de Septiembre de 2021, de https:// revistas.usat.edu.pe/index.php/educare/article/ view/450/1199?download=pdf

Inga, D., \& Aguirre, F. (2021). El enfoque de la educación virtual desde una perspectiva holístico frente a la pandemia del COVID-19. Revista Cátedra, 4(1), 81-97. Recuperado el 20 de Septiembre de 2021, de https://revistadigital.uce.edu.ec/index. php/CATEDRA/article/download/2727/3471/12263

Juca Maldonado, F. J. (2016). La educación a distancia, una necesidad para la formación de los profesionales. Revista Científica de la Universidad de Cienfuegos, 8(1), 106-111. Recuperado el 27 de Septiembre de 2021, de http://scielo.sld.cu/pdf/ rus/v8n1/rus15116.pdf

Leal Hoyos, Y. (2020). Educación virtual, retrospectiva y presente: una mirada reflexiva del docente del siglo XXI ante la crisis del Covid -19. Revista Arbitrada del CIEG, 45, 49-63. Recuperado el 18 de Septiembre de 2021, de https://www.grupocieg.org/archivos_revista/Ed.45(49-63)-Leal\%20 Yadel_articulo_id663.pdf
Organización de las Naciones Unidas para la Educación, la Ciencia y la Cultura - UNESCO. (2021). Organización de las Naciones Unidas para la Educación, la Ciencia y la Cultura - UNESCO. Recuperado el 20 de Septiembre de 2021, de https:// es.unesco.org/themes/educacion-superior/digital

Organización Mundial de la Salud - OMS. (27 de Abril de 2020). Organización Mundial de la Salud - OMS. Recuperado el 29 de Marzo de 2021, de https://www.who.int/es/news/item/27-04-2020who-timeline---covid-19

Palacios, J., Rodríguez, J., \& Forero, D. (2015). Educación y campus virtual, nuevos escenarios de formación. Revista Científica, 22, 97-110. Recuperado el 30 de Septiembre de 2021, de https://www. researchgate.net/publication/312270157_Educacion_y_Campus_Virtual_Nuevos_Escenarios_de_ Formacion/fulltext/587dd85808ae9275d4e960be/ Educacion-y-Campus-Virtual-Nuevos-Escenarios-de-Formacion.pdf?origin=publication_detail

Quezada, T. (2021). Educación virtual en la universidad en tiempos de covid-19. Espíritu Emprendedor TES, 5(1), 154-166. doi:https://orcid.org/00000002-0372-2071

Salgado García, E. (2015). La enseñanza y el aprendizaje en modalidad virtual desde la experiencia de estudiantes y profesores de posgrado. Tesis de Doctorado, Universidad Católica de Costa Rica, San José, Costa Rica. doi:https://www.aacademica.org/edgar.salgado.garcia/2

Urbina, S., \& Salinas, J. (2014). Campus virtuales: una perspectiva evolutiva y tendencias. RED. Revista de Educación a Distancia(42), 6-21. Recuperado el 30 de Septiembre de 2021, de https://www. redalyc.org/pdf/547/54731828002.pdf

Victorino, L. (2012). Retos de la universidad latinoamericana en el siglo XXI. El reto de la ESaD. Revista Calidad en la Educación Superior, 3(1), 1-23. doi:https://doi.org/10.22458/caes.v3i1.431

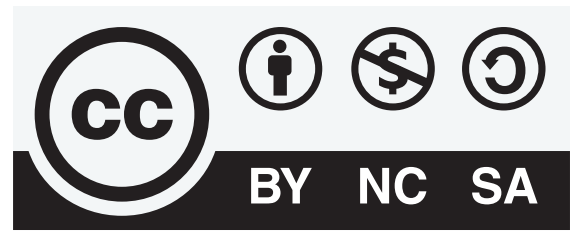

CREATIVE COMMONS RECONOCIMIENTO-NOCOMERCIAL-COMPARTIRIGUAL 4.0.

CITAR ESTE ARTICULO:

Pazmiño Peñafiel, E. S., Salcedo Aparicio, D., Ceballos Muñoz, V. I., \& Salcedo

Aparicio, P. R. (2021). Universidad Virtual. RECIMUNDO, 5(4), 12-21. https:// doi.org/10.26820/recimundo/5.(4).oct.2021.12-21 